\title{
Floristic composition of a conservation area in the Federal District of Brazil ${ }^{1}$
}

\author{
JULIANA SILVESTRE SILVA ${ }^{2,4}$ and JEANINE MARIA FELFILI ${ }^{3 \dagger}$
}

(received: March 19, 2012; accepted: August 10, 2012)

\begin{abstract}
Floristic composition of a conservation area in the Federal District of Brazil). Cerradão vegetation shares many species with savanna and forest areas and is one of the most vulnerable phytophysiognomies in the Cerrado (Brazilian savanna) biome. The floristic composition of the Cerradão Biological Reserve was examined between September/2007 and November/2008. A total of 282 species distributed among 194 genera and 75 families were encountered, demonstrating proportions of 0.91 herbaceous species and 0.54 shrub species for each tree species. Fabaceae, Asteraceae, Rubiaceae, Poaceae, Myrtaceae, Malpighiaceae, and Melastomataceae were the most species-rich families. Fully $72.3 \%$ of the species of this dystrophic cerradão were shared by cerrado and forest vegetations, while $60.43 \%$ were shared by other cerradão sites, although the largest proportion of species (91\%) were shared with cerrado sensu stricto. No species was found to be exclusive to this cerradão site, but approximately $95 \%$ of all species were native to the Cerrado biome.
\end{abstract}

Key words - APA Gama e Cabeça de Veado, cerradão, flora, species richness

\section{INTRODUCTION}

Cerradão is typified by a continuous arboreal layer more than $7 \mathrm{~m}$ tall, with 50 to $90 \%$ total canopy coverage; the shrub layer is generally dense and composed largely of sclerophyllous species, while there is only a reduced herbaceous layer. Lianas and some climbers are common, but epiphytes are rare (Ribeiro \& Walter 2008). Although cerradão constitutes a distinct cerrado (Brazilian savanna) phytophysiognomy, it does not have a distinctive flora, but rather a mix of floristic elements from cerrado sensu stricto and forests (Felfili 2002).

Very few studies have focused on the herbaceous/ subshrub flora of cerradão, as this is a predominantly arboreal phytophysiognomy. Additionally, although an extensive survey of the flora of the Gama and Cabeça de Veado Environmental ProtectionArea-EPA(in portuguese: Área de Proteção Ambiental-APA) was undertaken, the Cerradão Biological Reserve (in portuguese, Reserva Biológica-REBIO), also located in this APA, has not been examined in any detail. The present study was designed

1. Part of the Masters thesis of the first author, Programa de Pós Graduação em Botânica, Universidade de Brasília, Brasília, DF, Brazil.

2. Universidade de Brasília, Instituto de Ciências Biológicas, Departamento de Botânica, Caixa Postal 4457, 70910-970 Brasília, DF, Brazil.

$33^{\dagger}$ Universidade de Brasília, Faculdade de Tecnologia, Departamento de Engenharia Florestal, Laboratório de Manejo Florestal, CaixaPostal 04357, 70919-970 Brasília, DF, Brazil (in memoriam).

4._Corresponding author: julissilva@unb.br to contribute to future planning and management of the area and to our general knowledge of the mixed flora of this under-protected phytophysiognomy.

\section{MATERIAL AND METHODS}

The present study was undertaken in the Cerradão REBIO, a conservation area created by Federal Decree no 19.213/98 as an Area of Relevant Ecological Interest (in portuguese, Área de Relevante Interesse Ecológico - ARIE) and later transformed into a Biological Reserve (REBIO) by Federal Decree no $31.757 / 10$. This conservation area covers 54 ha (15 $51^{\prime} \mathrm{S}$ and $\left.47^{\circ} 49^{\prime} \mathrm{W}\right)$ and encompasses cerradão as well as dense cerrado vegetation. The regional climate is Tropical Altitudinal (Cwa by the Köppen classification system), with average temperatures in the coldest month below $18^{\circ} \mathrm{C}$, and above $22{ }^{\circ} \mathrm{C}$ in the warmest month; the average annual rainfall varies from $1,350 \mathrm{~mm}$ to $1,450 \mathrm{~mm}$. This REBIO is extremely important in protecting the water resources of the Federal District, as the region supplies approximately 50\% of the local aquifer recharge volume (Ibram 2010). The soils of the reserve are red latosols, similar to those found in other cerradão areas of the Gama and Cabeça de Veado EPA (Área de Preservação Ambiental - APA Gama e Cabeça de Veado) in the Federal District (Unesco 2002).

The floristic composition of the cerradão vegetation in the REBIO was examined by undertaking collections every two weeks during the period between September/2007 and November/2008. The cerradão vegetation (which differs from the adjacent dense cerrado vegetation by its more robust phytophysiognomy) within the reserve was surveyed by following a zigzag route through its entire area. 
Characteristics such as plant size and color and the presence of odors or latex were noted. Species identifications were carried out by comparisons with herbarium samples deposited in the University of Brasília (UB), Brasília Botanical Garden (HEPH), and IBGE (IBGE) herbaria, as well as by consulting the literature and taxonomists. In addition to the species collected during our survey, the floristic list includes species encounter during a phytosociological study of the arboreal layer of the same area undertaken by Silva (2009). Some of the species collected in the latter study were only encountered in their vegetative phase in the present survey and were not assigned collection numbers.

The data gathered in the present survey were compared to plant lists published by Mendonça et al. (2008) as the vascular flora of the Cerrado biome, by Proença et al. (2001) as the phanerogamic flora of the Federal District, and by Felfili et al. (2004) as the vascular flora of the Gama and Cabeça de Veado EPA, as well as in posterior publications as the phanerogamic flora of the Federal District series (Assis 2002, Carvalho-Silva \& Cavalcanti 2002, Lombardi 2002, Mendonça \& Amaral Junior 2002, Ribeiro \& Proença 2002, Chacon et al. 2003, Ferreira et al. 2003, Groppo 2003, Lima \& Pirani 2003, Pontes \& Mello-Silva 2003, Silva \& Valente 2003, França 2005, Silva \& Barros 2005, Fiaschi 2006, Martins \& Filgueiras 2006, Pastore \& Cavalcanti 2006, Dittrich et al. 2007, Groppo \& Pirani 2007).

The present survey considered: trees belonging to the arboreal layer, shrubs belonging to the shrub-layer, subshrubs, herbaceous plants, and climbers (lianas in general) composing the herbaceous layer, in order to allow comparisons with other floristic surveys, including Proença et al. (2001), Felfili et al. (2004), and Mendonça et al. (2008). Voucher specimens were deposited in the UB herbarium, and duplicates incorporate into the HEPH and UFMT (Universidade Federal do Mato Grosso) herbaria.

Synonyms were verified by consulting taxonomic revisions of specific groups as well as the databank sites of the Missouri Botanical Garden - $\mathrm{W}^{3}$ Tropicos (2008) and the International Plant Names Index - IPNI (2008). The botanical classification system followed the recommendations of the Angiosperm Phylogeny Group II (APG II 2003) for the phanerogams flora, and Smith et al. (2006) for the pteridoflora.

\section{RESULTS AND DISCUSSION}

We encountered 282 species belonging to 194 genera and 75 plant families in the Cerradão REBIO, of which 115 (40.78\%) were arboreal species, 62 (21.99\%) were shrubs, and $105(37.23 \%)$ made up the herbaceous stratum (54 herbs, 31 subshrubs, and 20 climbers) (table 1), resulting in ratios of 0.91 herbaceous species and 0.54 shrub species for each arboreal species. The data of Felfili et al. (2004) for cerradão sites in the Gama and Cabeça de Veado EPA in the Federal District (DF) yielded portions of 0.67 herbaceous species and 0.41 shrub species for each tree species; Proença et al. (2001) found 0.53 herbaceous plant species and 0.45 shrubs per arboreal species in cerradão sites in the Federal District; while Mendonça et al. (2008) reported portions of 1.05 herbaceous species and 0.61 shrubs for each arboreal layer species in cerradão sites in the Cerrado biome.

Table 1. The vascular flora of cerradão vegetation in the "REBIO Cerradão", Federal District, Brazil. Species identified by family, stratum, and phytophysiognomy according to the literature: Proença et al. 2001, Assis 2002, Carvalho-Silva \& Cavalcanti 2002, Lombardi 2002, Mendonça \& Amaral Junior 2002, Ribeiro \& Proença 2002, Chacon et al. 2003, Ferreira et al. 2003, Groppo 2003, Lima \& Pirani 2003, Pontes \& Mello-Silva 2003, Silva \& Valente 2003, Felfili et al. 2004, França 2005, Silva \& Barros 2005, Fiaschi 2006, Martins \& Filgueiras 2006, Pastore \& Cavalcanti 2006, Dittrich et al. 2007, Groppo \& Pirani 2007, Mendonça et al. 2008. (* = exotic/invasive; $\mathrm{S}=$ savanna sensu stricto; $\mathrm{S}(l s)$ = savanna sensu lato; $\mathrm{C}=$ cerradão $\mathrm{F}=$ forest; $\mathrm{GF}=$ gallery forest; $\mathrm{MF}=$ mesophytic/dry forest; $--=$ absent from the lists; Coll. $\mathrm{N} .=$ number of collection of JS Silva).

\begin{tabular}{llccc}
\hline Family & Species & Stratum & Phytophysiognomy & Coll. N. \\
\hline Acanthaceae & Justicia lanstyakii & Herb & S $(l s)$, S, GF & 16 \\
Alstroemeriaceae & Alstroemeria gardneri & Herb & S & 369 \\
Amaranthaceae & Gomphrena agrestis & Herb & S, C & 482 \\
& Pfaffia denudata & Herb & S & 218 \\
Anacardiaceae & Anacardium humile & Shrub & S, C & 4 \\
& Astronium fraxinifolium & Tree & S, C, GF, MF & 20 \\
Annonaceae & Annona crassiflora & Tree & S, C, GF & 236 \\
& A.tomentosa & Shrub & S, C, GF & 149 \\
& Duguetia furfuracea & Shrub & S, C, GF & 169 \\
& Guatteria sellowiana & Tree & F, GF & -- \\
& Xylopia aromatica & Tree & S, C, GF, MF & 69 \\
& & & & continue
\end{tabular}


continuation

\begin{tabular}{|c|c|c|c|c|}
\hline Family & Species & Stratum & Phytophysiognomy & Coll. N. \\
\hline & X. brasiliensis & Tree & $\mathrm{S}, \mathrm{C}, \mathrm{GF}$ & -- \\
\hline & $X$. sericea & Tree & C, F, GF, MF & 57 \\
\hline \multirow[t]{9}{*}{ Apocynaceae } & Aspidosperma macrocarpon & Tree & $\mathrm{S}, \mathrm{C}, \mathrm{MF}$ & 154 \\
\hline & A. spruceanum & Tree & $\mathrm{S}(l s), \mathrm{GF}, \mathrm{MF}$ & -- \\
\hline & A. subincanum & Tree & $\mathrm{F}, \mathrm{GF}, \mathrm{MF}$ & -- \\
\hline & A. tomentosum & Tree & $\mathrm{S}, \mathrm{C}, \mathrm{GF}, \mathrm{MF}$ & 501 \\
\hline & Ditassa obcordata & Shrub & $\mathrm{S}, \mathrm{C}, \mathrm{F}, \mathrm{GF}$ & 447 \\
\hline & Ditassa retusa & Climber & $\mathrm{S}, \mathrm{GF}$ & 614 \\
\hline & Hancornia speciosa & Tree & $\mathrm{S}, \mathrm{C}$ & 72 \\
\hline & Odontadenia lutea & Climber & $\mathrm{S}, \mathrm{C}, \mathrm{GF}, \mathrm{MF}$ & 209 \\
\hline & Prestonia coalita & Climber & $\mathrm{S}(l s), \mathrm{F}, \mathrm{GF}, \mathrm{MF}$ & 358 \\
\hline Aquifoliaceae & Ilex conocarpa & Tree & $\mathrm{S}, \mathrm{C}, \mathrm{GF}$ & 7 \\
\hline Araliaceae & Schefflera macrocarpa & Tree & $\mathrm{S}, \mathrm{C}, \mathrm{GF}$ & 172 \\
\hline \multirow[t]{3}{*}{ Arecaceae } & Allagoptera campestris & Shrub & $\mathrm{S}, \mathrm{C}$ & 203 \\
\hline & Syagrus comosa & Shrub & $\mathrm{S}, \mathrm{C}, \mathrm{GF}$ & 356 \\
\hline & S. flexиosa & Shrub & $\mathrm{S}, \mathrm{C}, \mathrm{GF}$ & 74 \\
\hline \multirow{23}{*}{ Asteraceae } & Achyrocline satureoides & Herb & $\mathrm{S}(l s), \mathrm{S}, \mathrm{GF}$ & 547 \\
\hline & Aspilia foliacea & Herb & $\mathrm{S}$ & 243 \\
\hline & Baccharis dracunculifolia & Subshrub & $\mathrm{S}(l s), \mathrm{GF}$ & 263 \\
\hline & B. salzmannii & Shrub & $\mathrm{S}(l s)$ & 120 \\
\hline & Campuloclinium megacephalum & Subshrub & $\mathrm{S}, \mathrm{F}, \mathrm{GF}$ & 384 \\
\hline & Chromolaena laevigata & Shrub & S, F, GF & 405 \\
\hline & C. leucocephala & Shrub & $\mathrm{S}$ & 177 \\
\hline & Echinocoryne holosericea & Herb & $\mathrm{S}$ & 534 \\
\hline & Elephanthopus mollis * & Herb & $\mathrm{S}, \mathrm{C}, \mathrm{F}, \mathrm{GF}, \mathrm{MF}$ & 527 \\
\hline & Emilia fosbergii* & Herb & $\mathrm{S}(l s)$ & 258 \\
\hline & Eremanthus glomerulatus & Shrub & $\mathrm{S}, \mathrm{C}, \mathrm{GF}$ & 159 \\
\hline & Gochnatia floribunda & Shrub & $\mathrm{S}(l s), \mathrm{S}, \mathrm{F}$ & 73 \\
\hline & Hoehnephytum trixoides & Subshrub & $\mathrm{S}$ & 46 \\
\hline & Icthyothere latifolia & Subshrub & $\mathrm{S}, \mathrm{F}$ & 368 \\
\hline & Lepidaploa aurea & Shrub & $\mathrm{S}, \mathrm{GF}$ & 99 \\
\hline & Lessingianthus compactiflorus & Subshrub & $\mathrm{S}, \mathrm{GF}$ & 362 \\
\hline & Piptocarpha macropoda & Tree & $\mathrm{S}, \mathrm{F}, \mathrm{GF}, \mathrm{MF}$ & 115 \\
\hline & P. rotundifolia & Tree & $\mathrm{S}, \mathrm{C}$ & 487 \\
\hline & Symphyopappus compressus & Subshrub & $\mathrm{S}, \mathrm{GF}$ & 292 \\
\hline & Trichogonia salviaefolia & Herb & $\mathrm{S}, \mathrm{GF}$ & 259 \\
\hline & Tridax procumbens $*$ & Herb & MF & 386 \\
\hline & Vernonia rubriramea & Shrub & $\mathrm{S}, \mathrm{C}, \mathrm{GF}$ & 87 \\
\hline & Wedelia bishopii & Herb & $\mathrm{S}$ & 344 \\
\hline Balanophoraceae & Langsdorffia hypogaea & Herb & $\mathrm{C}, \mathrm{F}, \mathrm{GF}$ & 517 \\
\hline \multirow[t]{8}{*}{ Bignoniaceae } & Adenocalymma pedunculatum & Shrub & S & 213 \\
\hline & Anemopaegma acutifolium & Subshrub & $\mathrm{S}$ & 519 \\
\hline & Cuspidaria sceptrum & Climber & $\mathrm{S}, \mathrm{C}, \mathrm{GF}, \mathrm{MF}$ & 176 \\
\hline & Fridericia platyphylla & Climber & $\mathrm{S}, \mathrm{C}, \mathrm{GF}$ & 381 \\
\hline & Handroanthus ochraceus & Tree & $\mathrm{S}, \mathrm{C}, \mathrm{GF}$ & 98 \\
\hline & H. serratifolius & Tree & $\mathrm{S}(l s), \mathrm{S}, \mathrm{F}, \mathrm{GF}, \mathrm{MF}$ & 59 \\
\hline & Jacaranda ulei & Subshrub & $\mathrm{S}, \mathrm{GF}$ & 103 \\
\hline & Zeyheria montana & Shrub & $\mathrm{S}, \mathrm{C}$ & 118 \\
\hline Blechnaceae & Blechnum australe supbsp. auriculatum & Herb & - & 274 \\
\hline Boraginaceae & Cordia truncata & Subshrub & $\mathrm{S}$ & 389 \\
\hline Bromeliaceae & Bromeliaceae sp. & Herb & - & 520 \\
\hline Burseraceae & Protium ovatum & Shrub & $\mathrm{S}, \mathrm{F}, \mathrm{GF}, \mathrm{MF}$ & 25 \\
\hline
\end{tabular}


continuation

\begin{tabular}{|c|c|c|c|c|}
\hline Family & Species & Stratum & Phytophysiognomy & Coll. N. \\
\hline Caryocaraceae & Caryocar brasiliense & Tree & $\mathrm{S}, \mathrm{C}$ & 95 \\
\hline \multirow[t]{3}{*}{ Celastraceae } & Cheiloclinium cognatum & Tree & GF & 277 \\
\hline & Plenckia populnea & Tree & $\mathrm{S}, \mathrm{C}, \mathrm{GF}$ & 205 \\
\hline & Salacia crassifolia & Shrub & $\mathrm{S}, \mathrm{GF}$ & 70 \\
\hline Chrysobalanaceae & Couepia grandiflora & Tree & $\mathrm{S}, \mathrm{C}, \mathrm{GF}, \mathrm{MF}$ & 502 \\
\hline \multirow[t]{3}{*}{ Clusiaceae } & Kielmeyera abdita & Shrub & $\mathrm{S}$ & 235 \\
\hline & K. coriacea & Tree & $\mathrm{S}, \mathrm{C}, \mathrm{GF}$ & 81 \\
\hline & K. speciosa & Tree & $\mathrm{S}, \mathrm{F}, \mathrm{GF}$ & -- \\
\hline \multirow[t]{2}{*}{ Combretaceae } & Terminalia argentea & Tree & $\mathrm{S}, \mathrm{C}, \mathrm{GF}$ & 77 \\
\hline & T. fagifolia & Tree & $\mathrm{S}, \mathrm{C}, \mathrm{GF}, \mathrm{MF}$ & 109 \\
\hline Connaraceae & Connarus suberosus & Tree & $\mathrm{S}, \mathrm{C}$ & 556 \\
\hline \multirow[t]{2}{*}{ Convolvulaceae } & Ipomoea procumbens & Climber & $\mathrm{S}$ & 328 \\
\hline & Merremia contorquens & Climber & $\mathrm{S}$ & 467 \\
\hline Cucurbitaceae & Cayaponia tayuya & Climber & $\mathrm{S}, \mathrm{F}, \mathrm{GF}$ & 343 \\
\hline Cunoniaceae & Lamanonia ternata & Tree & $\mathrm{S}(l s), \mathrm{C}, \mathrm{F}, \mathrm{GF}$ & -- \\
\hline \multirow[t]{3}{*}{ Cyperaceae } & Cyperus laxus & Herb & $\mathrm{S}(l s), \mathrm{GF}$ & 270 \\
\hline & Rhynchospora consanguinea & Herb & $\mathrm{S}, \mathrm{GF}$ & 347 \\
\hline & R. exaltata & Herb & $\mathrm{S}, \mathrm{C}, \mathrm{F}$ & 47 \\
\hline Dichapetalaceae & Tapura amazonica & Tree & $\mathrm{GF}, \mathrm{MF}$ & 603 \\
\hline Dilleniaceae & Davilla elliptica & Shrub & $\mathrm{S}, \mathrm{GF}$ & 155 \\
\hline Dioscoreaceae & Dioscorea dodecaneura & Climber & GF & 604 \\
\hline Ebenaceae & Diospyros hispida & Tree & $\mathrm{S}, \mathrm{C}, \mathrm{GF}, \mathrm{MF}$ & -- \\
\hline \multirow[t]{3}{*}{ Erythroxylaceae } & Erythroxylum daphnites & Tree & $\mathrm{S}(l s), \mathrm{S}, \mathrm{C}, \mathrm{GF}, \mathrm{MF}$ & -- \\
\hline & E. deciduum & Shrub & $\mathrm{S}, \mathrm{C}, \mathrm{F}, \mathrm{GF}$ & 607 \\
\hline & E. suberosum & Shrub & $\mathrm{S}, \mathrm{F}$ & 152 \\
\hline \multirow[t]{3}{*}{ Euphorbiaceae } & Dalechampia caperonioides & Herb & $\mathrm{S}$ & 342 \\
\hline & Manihot violacea & Shrub & $\mathrm{S}$ & 337 \\
\hline & Maprounea guianensis & Tree & $\mathrm{S}, \mathrm{C}, \mathrm{GF}, \mathrm{MF}$ & 5 \\
\hline \multirow[t]{24}{*}{ Fabaceae } & Acosmium dasycarpum & Tree & $\mathrm{S}, \mathrm{C}, \mathrm{GF}$ & 326 \\
\hline & Bauhinia cf. dumosa & Shrub & $\mathrm{S}$ & 425 \\
\hline & B. rufa & Subshrub & $\mathrm{S}, \mathrm{C}, \mathrm{GF}$ & 608 \\
\hline & Bowdichia virgilioides & Tree & $\mathrm{S}, \mathrm{C}, \mathrm{GF}, \mathrm{MF}$ & 557 \\
\hline & Calliandra dysantha & Subshrub & $\mathrm{S}(l s), \mathrm{S}, \mathrm{C}, \mathrm{GF}$ & 43 \\
\hline & Centrosema brasilianum & Climber & $\mathrm{S}(l s), \mathrm{GF}$ & 473 \\
\hline & Chamaecrista conferta & Subshrub & $\mathrm{S}$ & 85 \\
\hline & Copaifera langsdorffii & Tree & $\mathrm{S}, \mathrm{C}, \mathrm{GF}, \mathrm{MF}$ & 577 \\
\hline & Crotalaria flavicoma & Herb & $\mathrm{S}(l s), \mathrm{S}, \mathrm{GF}$ & 376 \\
\hline & C. cf. grandiflora & Shrub & $\mathrm{S}(l s), \mathrm{GF}$ & 418 \\
\hline & Dalbergia miscolobium & Tree & $\mathrm{S}, \mathrm{C}, \mathrm{GF}$ & 233 \\
\hline & Dimorphandra mollis & Tree & $\mathrm{S}, \mathrm{C}, \mathrm{GF}$ & 237 \\
\hline & Enterolobium gummiferum & Tree & $\mathrm{S}, \mathrm{C}, \mathrm{GF}$ & -- \\
\hline & Galactia boavista & Herb & $\mathrm{S}(l s)$ & 214 \\
\hline & G. grewiaefolia & Subshrub & $\mathrm{S}, \mathrm{C}$ & 372 \\
\hline & Hymenaea stigonocarpa & Tree & $\mathrm{S}, \mathrm{C}, \mathrm{GF}$ & 271 \\
\hline & Hymenolobium heringeranum & Tree & $\mathrm{F}, \mathrm{GF}, \mathrm{MF}$ & -- \\
\hline & Machaerium acutifolium & Tree & $\mathrm{S}, \mathrm{C}, \mathrm{GF}, \mathrm{MF}$ & 60 \\
\hline & M. орасит & Tree & $\mathrm{S}, \mathrm{C}, \mathrm{MF}$ & -- \\
\hline & Mimosa somnians & Subshrub & $\mathrm{S}(l s), \mathrm{S}, \mathrm{C}, \mathrm{GF}$ & 345 \\
\hline & M. velloziana $*$ & Shrub & $\mathrm{S}(l s), \mathrm{GF}$ & 599 \\
\hline & Periandra mediterranea & Shrub & $\mathrm{S}, \mathrm{C}, \mathrm{GF}$ & 91 \\
\hline & Piptadenia gonoacantha & Tree & $\mathrm{GF}, \mathrm{MF}$ & 611 \\
\hline & Plathymenia reticulata & Tree & S, C & $\begin{array}{c}452 \\
\text { contin }\end{array}$ \\
\hline
\end{tabular}


continuation

\begin{tabular}{|c|c|c|c|c|}
\hline Family & Species & Stratum & Phytophysiognomy & Coll. N. \\
\hline & Platypodium elegans & Tree & $\mathrm{S}(l s), \mathrm{S}, \mathrm{C}, \mathrm{GF}, \mathrm{MF}$ & 192 \\
\hline & Pterodon pubescens & Tree & $\mathrm{S}, \mathrm{C}, \mathrm{GF}$ & 112 \\
\hline & Stryphnodendron adstringens & Tree & $\mathrm{S}, \mathrm{C}$ & 490 \\
\hline & Stylosanthes guianensis & Herb & $\mathrm{S}(l s), \mathrm{S}$ & 114 \\
\hline & Tachigali vulgaris & Tree & $\mathrm{S}, \mathrm{C}, \mathrm{GF}$ & -- \\
\hline & Vatairea macrocarpa & Tree & $\mathrm{S}, \mathrm{C}, \mathrm{GF}$ & -- \\
\hline Gentianaceae & Voyria aphylla & Herb & $\mathrm{F}, \mathrm{GF}$ & 399 \\
\hline Hypericaceae & Vismia glaziovii & Tree & C, F, GF & 267 \\
\hline Icacinaceae & Emmotum nitens & Tree & $\mathrm{S}, \mathrm{C}, \mathrm{GF}$ & 83 \\
\hline \multirow[t]{5}{*}{ Lamiaceae } & Aegiphila lhotzkiana & Tree & $\mathrm{S}, \mathrm{C}, \mathrm{GF}$ & 244 \\
\hline & Hypenia brachystachys & Subshrub & $\mathrm{S}(l s), \mathrm{S}, \mathrm{C}, \mathrm{GF}$ & 538 \\
\hline & Hyptis lythroides & Subshrub & $\mathrm{S}$ & 127 \\
\hline & H. rubiginosa & Subshrub & $\mathrm{S}$ & 107 \\
\hline & H. villosa & Herb & $\mathrm{S}$ & 133 \\
\hline \multirow[t]{7}{*}{ Lauraceae } & Cassytha filiformis * & Herb & $\mathrm{S}$ & 364 \\
\hline & Endlicheria paniculata & Tree & GF & 254 \\
\hline & Nectandra reticulata & Tree & GF & -- \\
\hline & Ocotea aciphylla & Tree & GF & -- \\
\hline & O. diospyrifolia & Tree & $\mathrm{S}(l s), \mathrm{S}, \mathrm{GF}$ & 498 \\
\hline & O. pomaderroides & Tree & $\mathrm{S}(l s), \mathrm{S}, \mathrm{GF}$ & 9 \\
\hline & O. spixiana & Tree & $\mathrm{S}, \mathrm{C}, \mathrm{GF}$ & 21 \\
\hline \multirow[t]{2}{*}{ Loganiaceae } & Antonia ovata & Tree & $\mathrm{S}, \mathrm{C}, \mathrm{GF}$ & 497 \\
\hline & Strychnos pseudoquina & Tree & $\mathrm{S}, \mathrm{C}$ & 28 \\
\hline \multirow{2}{*}{ Loranthaceae } & Phthirusa ovata & Herb & $\mathrm{S}, \mathrm{GF}, \mathrm{MF}$ & 151 \\
\hline & Psittacanthus robustus & Herb & $\mathrm{S}, \mathrm{GF}$ & 226 \\
\hline \multirow[t]{3}{*}{ Lythraceae } & Cuphea spermacoce & Subshrub & $\mathrm{S}, \mathrm{C}$ & 242 \\
\hline & Diplusodon virgatus & Shrub & $\mathrm{S}, \mathrm{C}, \mathrm{GF}$ & 456 \\
\hline & Lafoensia pacari & Tree & $\mathrm{S}, \mathrm{C}, \mathrm{GF}, \mathrm{MF}$ & -- \\
\hline \multirow[t]{15}{*}{ Malpighiaceae } & Banisteriopsis anisandra & Climber & $\mathrm{S}(l s), \mathrm{S}, \mathrm{GF}$ & 78 \\
\hline & B. argyrophylla & Climber & $\mathrm{S}(l s), \mathrm{S}, \mathrm{C}, \mathrm{GF}, \mathrm{MF}$ & 359 \\
\hline & B. megaphylla & Shrub & $\mathrm{S}, \mathrm{GF}$ & 469 \\
\hline & B. stellaris & Climber & $\mathrm{S}, \mathrm{C}, \mathrm{GF}$ & 331 \\
\hline & Byrsonima crassifolia & Tree & $\mathrm{S}, \mathrm{C}$ & 79 \\
\hline & B. intermedia & Tree & $\mathrm{S}(l s), \mathrm{GF}$ & 183 \\
\hline & B. laxiflora & Tree & $\mathrm{S}(l s), \mathrm{S}, \mathrm{GF}, \mathrm{MF}$ & 150 \\
\hline & B. rotunda & Tree & -- & 221 \\
\hline & B. sericea & Tree & $\mathrm{S}(l s), \mathrm{S}, \mathrm{GF}$ & -- \\
\hline & B. verbascifolia & Tree & $\mathrm{S}, \mathrm{GF}$ & 145 \\
\hline & Heteropterys byrsonimifolia & Tree & $\mathrm{S}$ & 24 \\
\hline & H. pteropetala & Shrub & $\mathrm{S}, \mathrm{C}, \mathrm{F}, \mathrm{GF}$ & 330 \\
\hline & Janusia sp. & Climber & -- & 137 \\
\hline & Peixotoa goiana & Shrub & $\mathrm{S}$ & 478 \\
\hline & P. reticulata & Shrub & $\mathrm{S}$ & 543 \\
\hline \multirow[t]{5}{*}{ Malvaceae } & Eriotheca pubescens & Tree & $\mathrm{S}(l s), \mathrm{S}, \mathrm{C}, \mathrm{F}, \mathrm{GF}$ & -- \\
\hline & Pavonia rosa-campestris & Herb & $\mathrm{S}, \mathrm{C}$ & 117 \\
\hline & Pseudobombax longiflorum & Tree & $\mathrm{S}, \mathrm{CS}, \mathrm{GF}, \mathrm{MF}$ & 561 \\
\hline & Sida linifolia $*$ & Herb & $\mathrm{S}, \mathrm{GF}$ & 625 \\
\hline & Waltheria indica* & Shrub & $\mathrm{S}, \mathrm{GF}$ & 525 \\
\hline \multirow[t]{4}{*}{ Melastomataceae } & Leandra aurea & Shrub & $\mathrm{S}(l s), \mathrm{GF}$ & 336 \\
\hline & Miconia albicans & Tree & $\mathrm{S}, \mathrm{C}, \mathrm{GF}$ & 2 \\
\hline & M. burchellii & Tree & $\mathrm{S}, \mathrm{C}, \mathrm{GF}, \mathrm{MF}$ & 6 \\
\hline & M. cubatanensis & Tree & $\mathrm{S}, \mathrm{C}, \mathrm{GF}, \mathrm{MF}$ & 529 \\
\hline
\end{tabular}


continuation

\begin{tabular}{|c|c|c|c|c|}
\hline Family & Species & Stratum & Phytophysiognomy & Coll. N. \\
\hline & M. cuspidata & Tree & $\mathrm{S}(l s), \mathrm{S}, \mathrm{C}, \mathrm{GF}, \mathrm{MF}$ & 562 \\
\hline & M. fallax & Shrub & $\mathrm{S}, \mathrm{C}$ & 33 \\
\hline & M. ferruginata & Tree & $\mathrm{S}, \mathrm{C}, \mathrm{GF}$ & 8 \\
\hline & M. pepericarpa & Tree & $\mathrm{S}, \mathrm{GF}$ & 14 \\
\hline & M. pohliana & Tree & $\mathrm{S}, \mathrm{C}, \mathrm{GF}$ & 558 \\
\hline & Miconia sellowiana & Tree & $\mathrm{S}, \mathrm{C}, \mathrm{GF}, \mathrm{MF}$ & 573 \\
\hline & Ossaea congestiflora & Shrub & $\mathrm{S}, \mathrm{F}$ & 276 \\
\hline & Tibouchina stenocarpa & Tree & $\mathrm{S}, \mathrm{C}, \mathrm{F}, \mathrm{GF}$ & 426 \\
\hline Menispermaceae & Cissampelos pareira $*$ & Climber & GF & 609 \\
\hline Moraceae & Brosimum gaudichaudii & Shrub & $\mathrm{S}, \mathrm{C}$ & 66 \\
\hline Myristicaceae & Virola sebifera & Tree & $\mathrm{S}(l s), \mathrm{C}, \mathrm{GF}, \mathrm{MF}$ & 26 \\
\hline \multirow[t]{4}{*}{ Myrsinaceae } & Cybianthus densiflorus & Subshrub & $\mathrm{S}, \mathrm{F}, \mathrm{GF}$ & 126 \\
\hline & C. detergens & Subshrub & $\mathrm{S}(l s), \mathrm{S}, \mathrm{C}, \mathrm{GF}$ & 247 \\
\hline & Myrsine coriacea & Tree & $\mathrm{S}(l s), \mathrm{C}, \mathrm{GF}, \mathrm{MF}$ & 147 \\
\hline & M. guianensis & Tree & $\mathrm{S}(l s), \mathrm{S}, \mathrm{C}, \mathrm{GF}, \mathrm{MF}$ & 63 \\
\hline \multirow[t]{14}{*}{ Myrtaceae } & Blepharocalyx salicifolius & Tree & $\mathrm{S}, \mathrm{C}, \mathrm{GF}$ & 15 \\
\hline & Campomanesia pubescens & Shrub & $\mathrm{S}, \mathrm{GF}$ & 248 \\
\hline & Eugenia bracteata & Shrub & $\mathrm{S}(l s), \mathrm{S}, \mathrm{GF}$ & 174 \\
\hline & E. dysenterica & Tree & $\mathrm{S}, \mathrm{C}, \mathrm{GF}$ & -- \\
\hline & E. punicifolia & Shrub & $\mathrm{S}, \mathrm{C}, \mathrm{MF}$ & 451 \\
\hline & Myrcia cordifolia & Shrub & $\mathrm{S}, \mathrm{F}$ & 45 \\
\hline & M. nivea & Shrub & $\mathrm{S}(l s), \mathrm{S}, \mathrm{C}, \mathrm{GF}, \mathrm{MF}$ & 65 \\
\hline & M. rostrata & Tree & $\mathrm{S}$ & 105 \\
\hline & M. rubella & Subshrub & $\mathrm{S}(l s), \mathrm{S}$ & 27 \\
\hline & M. tomentosa & Tree & $\mathrm{S}, \mathrm{C}, \mathrm{GF}, \mathrm{MF}$ & 100 \\
\hline & Pimenta pseudocaryophyllus & Tree & $\mathrm{S}, \mathrm{GF}$ & 446 \\
\hline & Psidium firmum & Shrub & $\mathrm{S}, \mathrm{GF}$ & 595 \\
\hline & P. laruotteanum & Shrub & $\mathrm{S}, \mathrm{C}, \mathrm{GF}$ & 157 \\
\hline & Siphoneugena densiflora & Tree & $\mathrm{S}, \mathrm{C}, \mathrm{GF}$ & 17 \\
\hline \multirow[t]{5}{*}{ Nyctaginaceae } & Guapira areolata & Tree & GF & -- \\
\hline & G. graciliflora & Tree & $\mathrm{S}(l s), \mathrm{S}, \mathrm{C}, \mathrm{GF}$ & 12 \\
\hline & G. noxia & Tree & $\mathrm{S}, \mathrm{C}, \mathrm{GF}$ & 602 \\
\hline & G. opposita & Tree & $\mathrm{S}(l s), \mathrm{GF}, \mathrm{MF}$ & 23 \\
\hline & Neea theifera & Tree & $\mathrm{S}, \mathrm{C}, \mathrm{GF}$ & 161 \\
\hline \multirow[t]{4}{*}{ Ochnaceae } & Ouratea confertiflora & Shrub & $\mathrm{S}, \mathrm{C}$ & 121 \\
\hline & O. floribunda & Subshrub & $\mathrm{S}, \mathrm{GF}$ & 474 \\
\hline & O. hexasperma & Shrub & $\mathrm{S}, \mathrm{C}$ & 40 \\
\hline & O. riedeliana & Shrub & $\mathrm{S}$ & 180 \\
\hline Opiliaceae & Agonandra brasiliensis & Tree & $\mathrm{S}, \mathrm{C}, \mathrm{GF}, \mathrm{MF}$ & -- \\
\hline Orchidaceae & Oncidium varicosum & Herb & $\mathrm{GF}$ & 318 \\
\hline Oxalidaceae & Oxalis suborbiculata & Subshrub & $\mathrm{S}(l s), \mathrm{S}, \mathrm{C}, \mathrm{GF}$ & 206 \\
\hline Passifloraceae & Passiflora cerradensis & Climber & $\mathrm{C}, \mathrm{GF}$ & 610 \\
\hline Peraceae & Pera glabrata & Shrub & $\mathrm{S}(l s), \mathrm{S}, \mathrm{C}, \mathrm{GF}, \mathrm{MF}$ & 38 \\
\hline Piperaceae & Piper aduncum & Shrub & $\mathrm{C}, \mathrm{GF}$ & 273 \\
\hline \multirow[t]{8}{*}{ Poaceae } & Aristida pendula & Herb & $\mathrm{S}(l s)$ & 523 \\
\hline & Axonopus barbigerus & Herb & $\mathrm{S}$ & 524 \\
\hline & Echinolaena inflexa & Herb & $\mathrm{S}$ & 339 \\
\hline & Ichnanthus pallens & Herb & $\mathrm{S}, \mathrm{GF}$ & 288 \\
\hline & I. bambusiflorus & Herb & $\mathrm{S}, \mathrm{F}, \mathrm{GF}$ & 616 \\
\hline & Melinis minutiflora* & Herb & $\mathrm{S}, \mathrm{GF}$ & 287 \\
\hline & Mesosetum loliiforme & Herb & $\mathrm{S}$ & 620 \\
\hline & Panicum cervicatum & Herb & $\mathrm{S}, \mathrm{C}$ & 367 \\
\hline
\end{tabular}


continuation

\begin{tabular}{|c|c|c|c|c|}
\hline Family & Species & Stratum & Phytophysiognomy & Coll. N. \\
\hline & P. maximum $*$ & Herb & $\mathrm{S}(l s), \mathrm{GF}$ & 298 \\
\hline & P. selowii & Herb & $\mathrm{S}, \mathrm{C}, \mathrm{F}, \mathrm{GF}$ & 410 \\
\hline & Paspalum polyphyllum & Herb & $\mathrm{S}, \mathrm{GF}$ & 521 \\
\hline & Schizachyrium sanguineum * & Herb & $\mathrm{S}, \mathrm{GF}$ & 619 \\
\hline & S. tenerum & Herb & $\mathrm{S}, \mathrm{C}$ & 621 \\
\hline & Trachypogon sp.1 & Herb & -- & 623 \\
\hline & Trachypogon sp.2 & Herb & -- & 624 \\
\hline & Urochloa brizantha * & Herb & $\mathrm{S}(l s), \mathrm{GF}$ & 615 \\
\hline & U. decumbens $*$ & Herb & $\mathrm{S}$ & 264 \\
\hline \multirow[t]{2}{*}{ Polygalaceae } & Bredemeyera velutina & Shrub & $\mathrm{S}(l s), \mathrm{S}, \mathrm{GF}$ & 295 \\
\hline & Polygala violacea & Herb & $\mathrm{S}$ & 257 \\
\hline Proteaceae & Roupala montana & Tree & $\mathrm{S}, \mathrm{GF}$ & 239 \\
\hline \multirow[t]{18}{*}{ Rubiaceae } & Alibertia elliptica & Shrub & $\mathrm{S}(l s), \mathrm{S}, \mathrm{F}, \mathrm{GF}$ & 80 \\
\hline & A. macrophylla & Tree & $\mathrm{S}(l s), \mathrm{S}, \mathrm{C}, \mathrm{GF}, \mathrm{MF}$ & -- \\
\hline & A. sessilis & Tree & $\mathrm{S}(l s), \mathrm{S}, \mathrm{GF}, \mathrm{MF}$ & 144 \\
\hline & Amaioua guianensis & Tree & $\mathrm{S}(l s), \mathrm{GF}$ & -- \\
\hline & Chomelia ribesioides & Subshrub & $\mathrm{S}, \mathrm{GF}$ & 234 \\
\hline & Coccocypselum aureum & Herb & $\mathrm{S}(l s), \mathrm{S}, \mathrm{C}, \mathrm{GF}$ & 398 \\
\hline & Declieuxia fruticosa & Subshrub & $\mathrm{S}, \mathrm{GF}$ & 382 \\
\hline & Ferdinandusa elliptica & Tree & $\mathrm{S}, \mathrm{GF}$ & 18 \\
\hline & Galium noxium & Herb & GF & 265 \\
\hline & Palicourea marcgravii & Subshrub & $\mathrm{C}, \mathrm{GF}$ & 284 \\
\hline & P. officinalis & Subshrub & S, GF & 279 \\
\hline & P. rigida & Shrub & $\mathrm{S}, \mathrm{GF}$ & 241 \\
\hline & Psychotria hoffmannseggiana & Subshrub & $\mathrm{C}, \mathrm{GF}$ & 297 \\
\hline & P. prunifolia & Subshrub & $\mathrm{C}, \mathrm{GF}$ & 272 \\
\hline & P. sciaphila & Subshrub & $\mathrm{S}(l s), \mathrm{C}, \mathrm{GF}$ & 291 \\
\hline & Sabicea brasiliensis & Subshrub & $\mathrm{S}, \mathrm{GF}$ & 334 \\
\hline & Spermacoce poaya & Herb & $\mathrm{S}(l s), \mathrm{S}$ & 374 \\
\hline & Tocoyena formosa & Shrub & $\mathrm{S}, \mathrm{C}, \mathrm{GF}$ & 245 \\
\hline \multirow[t]{3}{*}{ Rutaceae } & Esenbeckia pumila & Shrub & $\mathrm{S}, \mathrm{GF}$ & 208 \\
\hline & Spiranthera odoratissima & Shrub & $\mathrm{S}, \mathrm{C}, \mathrm{GF}$ & 575 \\
\hline & Zanthoxylum rhoifolium & Tree & $\mathrm{S}(l s), \mathrm{C}, \mathrm{GF}$ & 606 \\
\hline \multirow[t]{2}{*}{ Salicaceae } & Casearia grandiflora & Shrub & $\mathrm{S}(l s), \mathrm{S}, \mathrm{C}, \mathrm{GF}, \mathrm{MF}$ & 346 \\
\hline & C. sylvestris & Shrub & $\mathrm{S}, \mathrm{C}, \mathrm{GF}$ & 552 \\
\hline \multirow[t]{4}{*}{ Sapindaceae } & Matayba guianensis & Tree & $\mathrm{S}, \mathrm{C}, \mathrm{GF}, \mathrm{MF}$ & 220 \\
\hline & Serjania erecta & Climber & $\mathrm{S}, \mathrm{F}, \mathrm{GF}$ & 375 \\
\hline & S. lethalis & Climber & $\mathrm{S}(l s), \mathrm{S}, \mathrm{GF}$ & 160 \\
\hline & S. ovalifolia & Climber & $\mathrm{S}(l s), \mathrm{GF}$ & 111 \\
\hline Sapotaceae & Pouteria ramiflora & Tree & $\mathrm{S}, \mathrm{C}, \mathrm{GF}$ & 576 \\
\hline Schizaeaceae & Anemia phyllitidis & Herb & $\mathrm{S}, \mathrm{GF}, \mathrm{MF}$ & 269 \\
\hline Simaroubaceae & Simarouba versicolor & Tree & $\mathrm{S}, \mathrm{C}, \mathrm{GF}, \mathrm{MF}$ & 108 \\
\hline Siparunaceae & Siparuna guianensis & Tree & $\mathrm{S}, \mathrm{C}, \mathrm{GF}, \mathrm{MF}$ & 261 \\
\hline Smilacaceae & Smilax brasiliensis & Climber & $\mathrm{S}, \mathrm{MF}$ & 190 \\
\hline Solanaceae & Solanum paniculatum * & Shrub & $\mathrm{S}, \mathrm{C}, \mathrm{GF}$ & 116 \\
\hline \multirow[t]{2}{*}{ Styracaceae } & Styrax camporum & Tree & $\mathrm{S}, \mathrm{C}, \mathrm{GF}, \mathrm{MF}$ & 251 \\
\hline & S. ferrugineus & Tree & $\mathrm{S}, \mathrm{C}, \mathrm{GF}$ & 39 \\
\hline \multirow{2}{*}{ Symplocaceae } & Symplocos mosenii & Tree & $\mathrm{S}(l s), \mathrm{S}, \mathrm{C}, \mathrm{GF}$ & 11 \\
\hline & S. nitens & Tree & $\mathrm{F}, \mathrm{GF}$ & 455 \\
\hline & Turnera lamiifolia & Herb & $\mathrm{S}(l s), \mathrm{S}$ & 488 \\
\hline \multirow{2}{*}{ Verbenaceae } & Lantana camara* & Shrub & $\mathrm{S}(l s), \mathrm{GF}, \mathrm{MF}$ & 296 \\
\hline & L. trifolia* & Shrub & $\mathrm{S}, \mathrm{MF}$ & 266 \\
\hline
\end{tabular}


continuation

\begin{tabular}{llccc}
\hline Family & Species & Stratum & Phytophysiognomy & Coll. N. \\
\hline \multirow{4}{*}{ Vochysiaceae } & Lippia rotundifolia & Shrub & S & 511 \\
& Stachytarpheta polyura & Herb & S $(l s)$, S, GF & 293 \\
& Callisthene major & Tree & S $(l s)$, S, GF, MF & 596 \\
& Qualea dichotoma & Tree & S $(l s)$, S, C, GF, MF & -- \\
& Q. grandiflora & Tree & S, C, GF & 283 \\
& Q. multiflora & Tree & S, C, GF & 104 \\
& Q. parviflora & Tree & S, C, GF & 56 \\
& Vochysia elliptica & Tree & S & -- \\
V. rufa & Tree & S, C, GF & -- \\
& V. thyrsoidea & Tree & S, C & 165 \\
& V. tucanorum & Tree & S $(l s)$, S, C, GF, MF & 49 \\
\hline
\end{tabular}

Higher proportions of arboreal species in cerradão sites as compared to herbaceous or shrub forms are not typical of the Cerrado biome as a whole, which consistently shows higher proportions of species in the herbaceous stratum. Mendonça et al. (2008) noted, however, that all of the subshrubs, climbers and lianas they identified as belonging to the herbaceous stratum could be considered as belonging to the shrub or even arboreal layer (as they were reasonably large, as in the case of woody vines). Other lists, including that obtained in the present study, have confirmed the predominance of the arboreal stratum in the cerradão phytophysiognomies.

Batalha \& Mantovani (2001) reported 148 species belonging to 113 genera and 45 families in an area of cerradão in São Paulo State, Brazil, with a proportion of herbaceous to woody species (0.8:1.0) similar to that of the present study. Comparisons of the richness values between any two areas, however, must take into consideration the collection frequencies and the flora types considered (vascular, or only phanerogams).

The families Fabaceae, Asteraceae, Rubiaceae, Poaceae, Malpighiaceae, Myrtaceae, Melastomataceae, Apocynaceae, and Vochysiaceae had the greatest species richness in the present study $(52.12 \%$ of the total number of species encountered). In relation to the numbers of genera, Fabaceae was again the most abundant family, followed by Asteraceae, Rubiaceae, Poaceae, Myrtaceae, and Bignoniaceae; Miconia was the most representative genus (nine species).

Rubiaceae, Myrtaceae, Fabaceae, Vochysiaceae, and Melastomataceae were also among the families demonstrating the greatest richnesses of woody species in areas of cerradão studied by Pereira-Silva et al. (2004); Fabaceae, Malvaceae, and Myrtaceae stood out among the cerradão families in Pantanal de Nhecolândia in Mato Grosso do Sul State (MS) (Salis et al. 2006); and Fabaceae and Vochysiaceae were the richest families identified in cerrado sensu stricto sites by Assunção \& Felfili (2004) and by Costa \& Araújo (2001), with Myrtaceae also showing high richness in the latter survey. Studies undertaken in forest areas demonstrated different results, however. The families Fabaceae, Melastomataceae, Myrtaceae, Rubiaceae, Apocynaceae, and Vochysiaceae were cited as demonstrating the greatest species richness in gallery forests, with important contributions also by Sapotaceae, Euphorbiaceae, and Moraceae (poorly represented in the present study) (Marimon et al. 2002, Silva Junior 2004); and Fabaceae, Bignoniaceae, Malvaceae, and Anacardiaceae were well represented in a semi-deciduous dry forest in Diorama, Goiás State (GO) (Santos-Diniz \& Souza 2011). It can therefore be seen that, in spite of the floristic differences between the phytophysiognomies, the richness values observed in the Cerradão REBIO were close to those reported for cerrado sensu stricto (Felfili et al. 1994, Costa \& Araújo 2001, Silva et al. 2002). According to Souza et al. (2010), the importance of Fabaceae in terms of species richness in the Cerrado biome is due to the capacity of many of its species to fix nitrogen, enabling them to growth in poor and degraded soils.

Lopes (1984) noted that plant distributions are controlled by very basic factors such as the climate, soils, and the availability of water and nutrients, but can also be greatly influenced by latitude, burning frequencies, water table depth, animal pasturing, and anthropogenic factors - which reinforces the importance of floristic and phytosociological studies at many different sites, as each area would be expected to have specific physical conditions and therefore unique population structures (Santos-Diniz \& Souza 2011).

The data published in the three plant lists used for comparisons in the present work, in the flora of the 
Federal District, and in floristic and phytosociological studies undertaken in both savanna and cerrado forest phytophysiognomies (Felfili et al. 1994, Silva Junior et al. 1998, Marimon et al. 2002, Silva et al. 2002, Assunção \& Felfili 2004, Pereira-Silva et al. 2004, Silva Junior 2004, Balduino et al. 2005, Santiago et al. 2005, Salis et al. 2006) indicated that $91 \%$ of the fully-identified species encountered in the REBIO cerradão were common to cerrado areas, while $79.5 \%$ were common to forest sites. The species commonly encountered in both formations represent $72.3 \%$ of the total, while only $60.43 \%$ of the fully identified species of the REBIO were reported in other cerradão surveys. No single species was exclusive to the cerradão phytophysiognomy.

The elevated species richness identified in the study area (with almost $95 \%$ species being native to the Cerrado biome) indicates the importance of this conservation area, for it not only protects a significant area of cerradão vegetation (a phytophysiognomy poorly represented in other designated conservation areas) but also conserves species such as Copaifera langsdorffi (copaiba), Pterodon pubescens (sucupira-branca), Caryocar brasiliense (pequizeiro), Eugenia dysenterica (cagaiteira), Vochysia thyrsoidea (gomeira), Vochysia tucanorum (pau-doce), Pseudobombax longiflorum (embiruçu), Dalbergia miscolobium (jacarandá), Aspidosperma spp. (perobas), and Tabebuia spp. (ipês) that have been designated as protected elements of the Ecological Heritage of the Federal District (Felfili \& Santos 2002).

The results obtained in the present study enrich our knowledge of the cerradão flora and can serve as subsidies for the elaboration of management programs for this conservation area (REBIO).

\section{REFERENCES}

APG II. 2003. An update of the Angiosperm Phylogeny Group classification for the orders and families of flowering plants: APG II. Botanical Journal of the Linnean Society 141:399-436.

Assis MC. 2002. Alstroemeriaceae. In Flora do Distrito Federal, Brasil (TB Cavalcanti, AE Ramos, orgs.). Embrapa Recursos Genéticos e Biotecnologia, Brasília, v.2, p.19-32.

Assunção SL, Felfili JM. 2004. Fitossociologia de um fragmento de cerrado sensu stricto na APA do Paranoá, DF, Brasil. Acta Botanica Brasilica 18:903-909.

Balduino APC, Souza AL, Meira Neto JAA, Silva AF, Silva Junior MC. 2005. Fitossociologia e análise comparativa da composição florística do cerrado da flora de Paraopeba-MG. Revista Árvore 29:25-34.
Batalha MA, Mantovani W. 2001. Floristic composition of the Cerrado in the Pé-de-Gigante Reserve (Santa Rita do Passa Quatro, Southeastern, Brazil). Acta Botanica Brasilica 15:289-304.

Carvalho-Silva M, Cavalcanti TB. 2002. Piperaceae. In Flora do Distrito Federal, Brasil (TB Cavalcanti, AE Ramos, orgs.). Embrapa Recursos Genéticos e Biotecnologia, Brasília, v.2, p.91-124.

Chacon RG, Yamamoto K, Cavalcanti TB. 2003. Ochnaceae. In Flora do Distrito Federal, Brasil (TB Cavalcanti, AE Ramos, orgs.). Embrapa Recursos Genéticos e Biotecnologia, Brasília, v.3, p.205-226.

Costa AA, Araújo GM. 2001. Comparação da vegetação arbórea de cerradão e de cerrado na Reserva do Panga, Uberlândia, Minas Gerais. Acta Botanica Brasilica 15:63-72.

Dittrich VAO, Heringer G, Salino A. 2007. Blechnaceae. In Flora do Distrito Federal, Brasil (TB Cavalcanti, org.). Embrapa Recursos Genéticos e Biotecnologia, Brasília, v.6, p.89-108.

Felfili JM. 2002. Padrões de diversidade do Cerrado do Centro-Oeste brasileiro. In Biodiversidade, conservação e uso sustentável da flora do Brasil (EL Araújo, AN Moura, ESB Sampaio, LMS Gestinari, JMT Carneiro, eds.). Universidade Federal Rural de Pernambuco Imprensa Universitária, Recife, p.58-61.

Felfili JM, Santos AAB. 2002. Legislação Ambiental: APA Gama e Cabeça de Veado. Universidade de Brasília, Faculdade de Tecnologia, Departamento de Engenharia Florestal, Brasília.

Felfili JM, Haridasan M, Mendonça RC, Filgueiras TS, Silva Junior MC, Rezende AV. 1994. Projeto biogeografia do Bioma Cerrado: vegetação e solos. Caderno de Geociências 12:75-166.

Felfili JM, Mendonça RC, Munhoz CBR, Fagg CW, Pinto JRR, Silva Junior MC, Sampaio JC. 2004. Vegetação e flora da APA Gama e Cabeça de Veado. In Flora e diretrizes ao plano de manejo da APA Gama e Cabeça de Veado (JM Felfili, AAB Santos, JC Sampaio, orgs.). Universidade de Brasília, Faculdade de Tecnologia, Departamento de Engenharia Florestal, Brasília, p.7-16.

Ferreira JN, Aquino FG, Okano RC, Proença CEB. 2003. Celastraceae. In Flora do Distrito Federal, Brasil (TB Cavalcanti, AE Ramos, orgs.). Embrapa Recursos Genéticos e Biotecnologia, Brasília, v.3, p.139-150.

Fiaschi P. 2006. Oxalidaceae. In Flora do Distrito Federal, Brasil (TB Cavalcanti, org.). Embrapa Recursos Genéticos e Biotecnologia, Brasília, v.5, p.91-106.

França F. 2005. Vochysiaceae. In Flora do Distrito Federal, Brasil (TB Cavalcanti, AE Ramos, orgs.). Embrapa Recursos Genéticos e Biotecnologia, Brasília, v.4, p.77-106. 
Groppo M. 2003. Aquifoliaceae. In Flora do Distrito Federal, Brasil (TB Cavalcanti, AE Ramos, orgs.). Embrapa Recursos Genéticos e Biotecnologia, Brasília, v.3, p.49-62.

Groppo M, Pirani JR. 2007. Rutaceae. In Flora do Distrito Federal, Brasil (TB Cavalcanti, org.). Embrapa Recursos Genéticos e Biotecnologia, Brasília, v.6, p.63-86.

Ibram. 2010. Proposta de mudança de categoria: Área de Relevante Interesse Ecológico do Cerradão - Reserva Biológica do Cerradão. Governo do Distrito Federal, Brasília.

Lima LR, Pirani JR. 2003. Burseraceae. In Flora do Distrito Federal, Brasil (TB Cavalcanti, AE Ramos, orgs.). Embrapa Recursos Genéticos e Biotecnologia, Brasília, v.3, p.125-138.

Lombardi JA. 2002. Hippocrateaceae. In Flora do Distrito Federal, Brasil (TB Cavalcanti, AE Ramos, orgs.). Embrapa Recursos Genéticos e Biotecnologia, Brasília, v.2, p.73-90.

Lopes AS. 1984. Solos sob "cerrado": características, propriedades e manejo. 2nd ed. Associação Brasileira para Pesquisa da Potassa e do Fosfato, Piracicaba.

Marimon BS, Felfili JM, Lima ES. 2002. Floristics and phytosociology of the gallery forest of the Bacaba Stream, Nova Xavantina, Mato Grosso, Brazil. Edinburgh Journal of Botany 59: 303-318.

Martins RC, Filgueiras TS. 2006. Arecaceae. In Flora do Distrito Federal, Brasil (TB Cavalcanti, org.). Embrapa Recursos Genéticos e Biotecnologia, Brasília, v.5, p.45-82.

Mendonça JO, Amaral Junior A. 2002. Erythroxylaceae. In Flora do Distrito Federal, Brasil (TB Cavalcanti, AE Ramos, orgs.). Embrapa Recursos Genéticos e Biotecnologia, Brasília, v.2, p.53-71.

Mendonça RC, Felfili JM, Walter BMT, Silva Junior MC, Rezende AB, Filgueiras TS, Nogueira PE, Fagg CW. 2008. Flora vascular do bioma Cerrado: checklist com 12.356 espécies. In Cerrado: ecologia e flora (SM Sano, SP Almeida, JF Ribeiro, eds.). Embrapa Cerrados/ Embrapa Informação Tecnológica, Brasília, v.2, p.4211279.

Missouri Botanical Garden - W3 Tropicos. 2008. http://www. tropicos.org (accessed 2008 Nov 07).

Pastore JFB, Cavalcanti TB. 2006. Polygalaceae. In Flora do Distrito Federal, Brasil (TB Cavalcanti, org.). Embrapa Recursos Genéticos e Biotecnologia, Brasília, v.5, p.107-180.

Pereira-Silva EFL, Santos JE, Kageyama PY, Hardt E. 2004. Florística e fitossociologia dos estratos arbustivo e arbóreo de um remanescente de cerradão em uma unidade de conservação do Estado de São Paulo. Revista Brasileira de Botânica 27: 533-544.
Pontes AF, Mello-Silva R. 2003. Annonaceae. In Flora do Distrito Federal, Brasil (TB Cavalcanti, AE Ramos, orgs.). Embrapa Recursos Genéticos e Biotecnologia, Brasília, v.3, p.19-48.

Proença CEB, Munhoz CBR, Jorge CL, Nóbrega MGG. 2001. Listagem e nível de proteção das espécies de fanerógamas do Distrito Federal, Brasil. In Flora do Distrito Federal I (TB Cavalcanti, AE Ramos, eds.). Embrapa Recursos Genéticos e Biotecnologia, Brasília, p.89-359.

Ribeiro JF, Walter BMT. 2008. As principais fitofisionomias do bioma Cerrado. In Cerrado: ecologia e flora (SM Sano, SP Almeida, JF Ribeiro, eds.). Embrapa Cerrados/ Embrapa Informação Tecnológica, Brasília, 2nd ed., p.151-212.

Ribeiro MF, Proença CEB. 2002. Caryocaraceae. In Flora do Distrito Federal, Brasil (TB Cavalcanti, AE Ramos, orgs.). Embrapa Recursos Genéticos e Biotecnologia, Brasília, v.2, p.33-38.

Salis SM, Assis M, Crispim SMA, Casagrande JC. 2006. Distribuição e abundância de espécies arbóreas em cerradões no Pantanal, Estado do Mato Grosso do Sul, Brasil. Revista Brasileira de Botânica 29:339-352.

Santiago J, Silva Junior MC, Lima LC. 2005. Fitossociologia da regeneração arbórea na mata de galeria do Pitoco (IBGE-DF), seis anos após fogo acidental. Scientia Forestalis 67:64-77.

Santos-Diniz VS, Sousa TD. 2011. Levantamento florístico e fitossociológico de mata seca semidecídua em área de Reserva Legal do município de Diorama, região oeste de Goiás, Brasil. Enciclopédia Biosfera 7:1-17.

Silva JSS. 2009. Diversidade alfa, florística e fitossociologia na ARIE do Cerradão, na APA Gama e Cabeça de Veado, DF. Dissertação de mestrado, Universidade de Brasília, Brasília.

Silva LO, Costa DA, Espírito-Santo Filho K, Ferreira HD, Brandão D. 2002. Levantamento florístico e fitossociológico em duas áreas de cerrado sensu stricto no Parque Estadual da Serra de Caldas Novas, Goiás. Acta Botanica Brasilica 16:43-53.

Silva MRP, Barros ICL. 2005. Schizaeaceae. In Flora do Distrito Federal, Brasil (TB Cavalcanti, AE Ramos, orgs.). Embrapa Recursos Genéticos e Biotecnologia, Brasília, v.4, p.217-248.

Silva NMF, Valente MC. 2003. Combretaceae. In Flora do Distrito Federal, Brasil (TB Cavalcanti, AE Ramos, orgs.). Embrapa Recursos Genéticos e Biotecnologia, Brasília, v.3, p.151-170.

Silva Junior MC. 2004. Fitossociologia e estrutura diamétrica da Mata de galeria do Taquara, na Reserva Ecológica do IBGE, DF. Revista Árvore 28:419-428.

Silva Junior MC, Felfili JM, Nogueira PE, Rezende AB. 1998. Análise florística das matas de galeria no Distrito Federal. In Cerrado: matas de galeria (JF Ribeiro, ed.). Embrapa Cerrados, Planaltina, p.53-84. 
Smith AR, Pryer KM, Schuettpelz E, Korall P, Schneider H, Wolf PG. 2006. A classification for extant ferns. Taxon 55:705-731.

Souza PB, Saporetti Junior AW, Soares MP, Viana RHO, Camargos VL, Meira Neto JAA. 2010. Florística de uma área de cerradão na Floresta Nacional de Paraopeba Minas Gerais. Cerne 16:86-93.
The Internacional Plant Names Index - IPNI. 2008. http:// www.ipni.org (accessed 2008 Nov 07).

Unesco. 2002. Vegetação do Distrito Federal: tempo e espaçoUma avaliação multitemporal da perda de cobertura vegetal no DF e da diversidade florística da Reserva da Biosfera do Cerrado - Fase I. 2nd ed., Unesco, Brasília. 
